\title{
Does the pulvinar-LP complex contribute to motor programming?
}

\author{
J. Cudeiro, E González, R. Pérez, J.M. Alonso and C. Acuña
}

\begin{abstract}
Extracellular unit recording studies in the pulvinar lateral posterior complex (Pui-LP) of behaving monkeys have shown a response property not previously reported. In monkeys performing aimed arm reaching movements towards frontally located targets some cells showed a change in activity beginning $495 \pm 84 \mathrm{~ms}$ before the onset of the reaching movement. This change in frequency precedes that observed in primary motor and parietal posterior cortex for reaching movements. These findings seem to indicate the involvement of the Pul-LP in motor functions and suggest its possible contribution to motor programming.
\end{abstract}

Key words: Pulvinar lateral posterior complex; Behaving monkey; Extracellular recording; Motor programming; Reaching movement; Reaching cells

In the past few years morphological and physio- logical techniques have been used to understand the role of the pulvinar lateral posterior complex (Pul- LP). Although it forms the largest mass in the primate thalamus, its function is not yet clear. Pul-LP activity has been related to a number of functions such as vision, visuomotor, motor, auditive and attentive functions ${ }^{1-3,7-9,22,24,25,28}$.

The simultaneous phylogenetic development of the association neocortical areas and the Pul-LP ${ }^{5,14}$ and their reciprocal anatomical connections ${ }^{15}$ suggest a close functional relationship and it is reasonable to assume that the Pul-LP may play some role in higher level integrative associative functions ${ }^{1,21,23}$.

Recent studies in our laboratory have focused on the properties of reaching neurons previously described in the Pul-LP ${ }^{1,2}$. In the course of these studies we have found that some PuI-LP reaching cells increased their firing rate well before the earliest change reported for primary motor (MI) ${ }^{10,11}$ and posterior parietal cortex (area 5) ${ }^{16}$ reaching cells. In what follows a description of these cells is presented.

The experiments were carried out in 4 behaving Macaca nemestrina monkeys. A detailed description of our experimental and data analysis procedures have been published elsewhere ${ }^{2}$. Briefly, single unit activity was recorded from the Pul-LP by means of Pt-Ir glass-coated metal microelectrodes advanced through the intact dura. Monkeys were trained to perform arm reaching movements directed to 4 spatially separated targets situated on a frontal panel (Fig. 1). A trial began when the monkey pressed a lever key situated at its side; this lever turned on 1 of 4 LEDs in a random fashion. After a short random period the LED dimmed and the monkey had to release the key and press with its finger tips a switch below it. An attentive visual fixation of the LED was required in order to detect the dimming. The EOG was continuously monitored and eye movements were not allowed during the fixation period of the task. Recording of unitary extracellular activity was made while the animal was performing the task. Additionally, qualitative explorations were made; muscle palpation, brushing the skin, passive limb movements, and presentation of small pieces of food which the monkey had to reach for. At the end of the experiments the animals were sacrificed with a pentobarbital overdose, and the brains processed for the reconstruction of the penetrations.

The activity of 766 cells were recorded in 109 penetrations (unpublished data, ref. 3). Six cells out of these 766 increased their firing rate well before the beginning of the reaching movement, i.e. $495 \pm 84 \mathrm{~ms}$ before the monkey released the key. Fig. 1 shows an example of such cells. These neurons, located in the lateral posterior $(n=2)$ and oral pulvinar $(n=4)$, have the following additional characteristics: (1) they significantly increase their firing rate during reaching movements $(\mathrm{P}<0.05$, Student's $t$ and Duncan tests) but the increase is independent of the starting point, amplitude or direction of the movement: the response is related only to the act of reaching itself; (2) they are not driven by passive stimulation of the limb; and (3) they show dependence on states of attention or motivation of the animal (a general characteristic of Pul-LP cells). The activation shown by these cells seems to be related to the intention to execute a reaching movement towards a previously established target. The cells were not driven by neither spontaneous nor passive movements, but they significantly changed their activity with intentional movements. Qualitatively, we were able to observe that similar responses were elicited when the monkey 
projected its arm towards small pieces of food or objects that strongly attracted its attention. This neuronal population would be activated upon convergence of several sensory and limbic inputs, whereas the sensory inputs alone are irrelevant. Connections between the Pul-LP and several sensory ${ }^{9,27}$ and limbic systems ${ }^{4,20}$ and primary motor cortex $\mathrm{m}$ have been reported. These cell activity changes cannot be related to eye movements because they were not allowed during the fixation period of the task. Visual fixation cannot be involved in their responses either, because in those trials with a long visual fixation period and no hand projection there was no demonstrated effect on their discharge. On the other hand, cell activity changes do not seem to signal the eye position within the orbital cavity because no differences were found when the animal performed the task on each of the 4 different targets on the frontal panel.

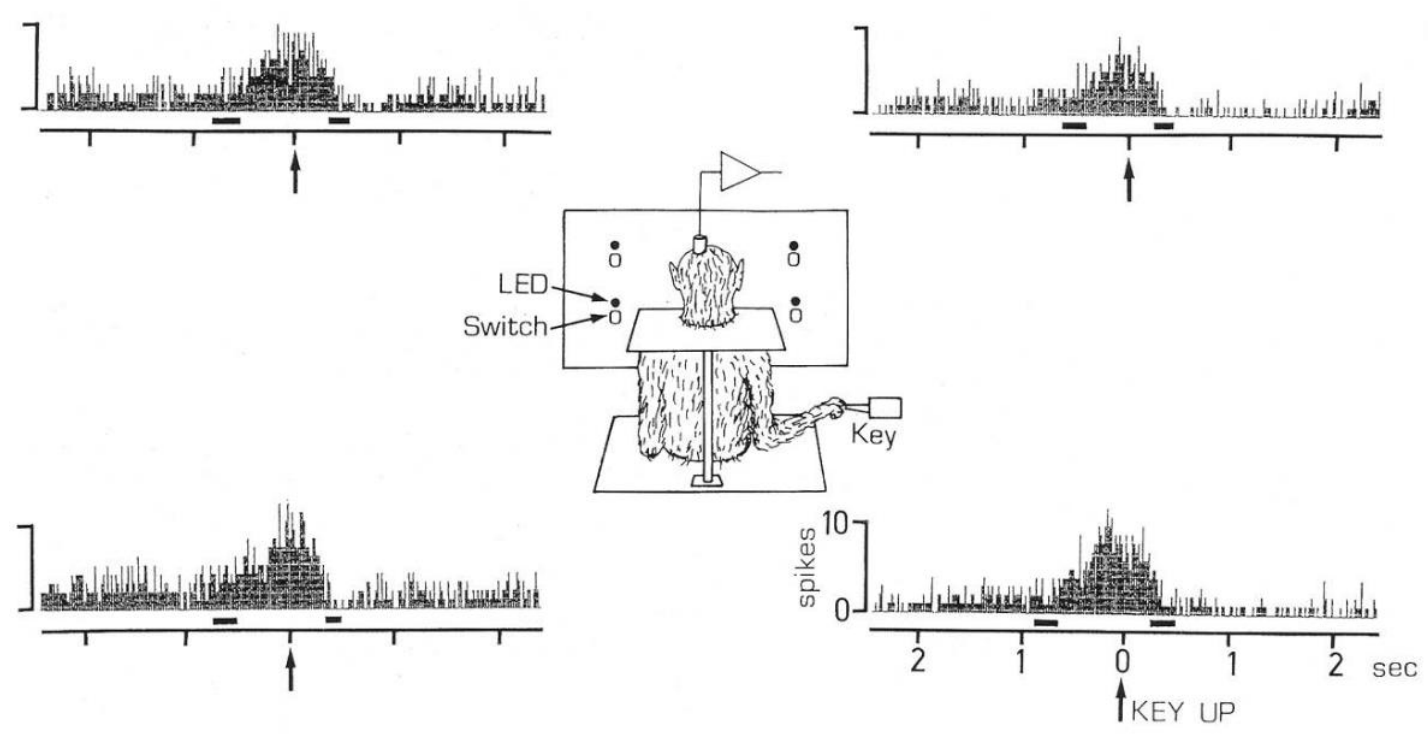

Fig. 1. Example of a cell recorded from the oral pulvinar. The 4 histograms show the cell discharge for 4 directions of reaching movements (towards each 1 of the 4 switches; about 5 trials each). The 2 lines under the spike histograms show the LED dimming (left) and the moment the monkey presses the panel switch (right). Arrows signal key releasing. The central inset shows the monkey while performing the task. There is no significant difference among the 4 spike histograms and the discharge starts in all instances well before the beginning of the reaching movement (key up).

Georgopoulos et al. ${ }^{10,11}$ found that the MI earliest change in activity takes place $280 \mathrm{~ms}$ before the beginning of the reaching movement. Kalaska et a1. ${ }^{16}$, using a similar experimental paradigm, showed that the onset of activity in the posterior parietal cortex (area 5) was delayed in relation to MI. In one monkey we made recordings in the parietal area 5 while performing the task and the results matched those of Kalaska et a1. ${ }^{16}$ (unpublished results, ref. 3), thus proving the validity of the task to establish comparisons. After comparing our Pul-LP results with those from Georgopoulos et al. ${ }^{10,11}$ we found that 6 cells, the object of this report, preceded the ones in MI in their activation. This early Pul-LP activity may provide information to MI about an immediate movement before activity in MI starts.

The typical behavior of these cells, i.e. no relation to the metrics of the movement and early activation, suggests that they may inform MI about the immediate execution of an intentional movement independently of its metrics. Cortical structures related to the programming of motor acts have been described 6,12,13,18,26, but no subcortical structures have previously been associated with motor programming in a similar way. In the supplementary motor area (SMA) and in area 6, cells that change their activity during preparation for specific movements have been described ${ }^{12}$. The cells involved in motor programming in SMA are less dependent on visual or other external cues than are neurons in area 6 (ref. 6). The changes in activity of some of these cells during the preparatory period are dearly related to the direction of arm movements required to reach for a visible target ${ }^{12}$. This dependency of cell activity on the direction of the movement establishes a difference with the Pul-LP cells described here, which are independent of the metrics of the movement.

The fact that the neocortex and the Pul-LP have developed phylogenetically along with the animal's ability to perform complex aimed movements and higher level integrative functions, the similarity of cellular activity patterns between SMA and premotor cortex, and our data, suggest that the Pul-LP 
complex is a subcortical structure which may be related to the programming of intentional movements. The described anatomical connections be- tween those cortical areas and the Pul-LP ${ }^{15,17}$ support the idea that both structures may be closely related in relation to the role they play in the programming of aimed movements towards reachable objects.

We acknowledge the Ayudas PR83-2100 of the CAICYT, and the Xunta de Galicia 1987, Spain for partially supporting this work. J.C. was a Fellow of the FISS 1986-1988, Spain.

\section{References}

1 Acuña, C., González, E and Domfnguez, R., Sensorimotor unit activity related to intention in the pulvinar of behaving Cebus apella monkeys, Exp. Brain Res., 52 (1983) 411-422.

2 Acuña, C., Cudeiro, J. and González, E, Lateral posterior (Lp) and pulvinar unit activity related to intentional upper limb movements directed to spatially separated targets in behaving Macaca nemestrina monkeys, Rev. Neurol., 142 (1986) 354-361.

3 Acuña, C., Cudeiro, J., González, F., Alonso, J.M. and Pérez, R., Lateroposterior and pulvinar reaching cells. Comparison with parietal area 5a. A study in Macaca nemestrina monkeys, in preparation.

4 Aggleton, J.P., Desimone, R. and Mishkin, M., The origin, course and termination of the hippocampo-thalamic projections in the macaque, J. Comp. Neurol., 243 (1986) 409-421.

5 Armstrong, E., A quantitative comparison of the hominoid thalamus-IV posterior association nuclei. The pulvinar and lateroposterior nucleus, Am. J. Physiol. Anthropol., 55 (1981) 369-383.

6 Brinkman, C., Comparison of the activity of neurones in the supplementary motor and premotor areas associated with self-paced movements in the monkey, Exp. Brain Res., 58 (1985) A7.

7 Crighel, E. and Kreindler, A., Relations between the pulvinar lateralis posterior complex and the neural systems involved in the organization of the motor pattern, Acta Physiol. Acad. Sci. Hung., 48 (1976) 399-406.

8 Crighel, E., The role of the pulvinar lateralis posterior complex of the thalamus in the organization of motor patterns. In K. Lissak (Ed.), Neural and Neurohormonal Organization of Motivated Behavior, Akad. Kiado, Buda- pest, 1978.

9 Chalupa, L.M., A review of cat and monkey studies implicating the pulvinar in visual functions, Behav. Biol., 20 (1977) $149-167$.

10 Georgopoulos, A.P., Kalaska, J.F., Caminiti, R. and Massey, J.T., On the relations between the direction of twodimensional arm movements and cell discharge in primate motor cortex, J. Neurosci., 2 (1982) 1527-1537.

11 Georgopoulos, A.P., Caminiti, R., Kalaska, J.E and Massey, J.T., Spatial coding of movement: a hypothesis concerning the coding of movement direction by motor cortical populations, Exp. Brain Res., Suppl. 7 (1983) 327-336.

12 Godschalk, M., Lemon, R.N., Nijs, H.G.T. and Kuypers, H.G.J.M., Behavior of neurons in monkey peri-arcuate and precentral cortex before and during visually guided arm and hand movements, Exp. Brain Res., 44 (1981) 113-116.

13 Goldberg, G., Supplementary motor area structure and function: review and hypothesis, Behav. Brain Sci., 8 (1985) 567-616.

14 Harting, J.K., Hall, W.C. and Diamond, I.J., Evolution of the pulvinar, Brain Behav. EvoL, 6 (1972) 424-452.

15 Jurgens, U., Efferent connections of the supplementary motor area, Exp. Brain Res., 58 (1985) A1.

16 Kalaska, J.E, Caminiti, R. and Georgopoulos, A.P., Cortical mechanisms related to directions of two-dimensional arm movements: relations in parietal area 5 and comparison with motor cortex, Exp. Brain Res., 51 (1983) 247-260

17 Kievit, J. and Kuypers, H.G.J.M., Organization of the thalamocortical connections to the frontal lobe in the rhesus monkey, Exp. Brain Res., 29 (1977) 229-322.

18 Kornhuber, H.H., Mechanisms of voluntary movement. In W. Prinz and A.E Sanders (Eds.), Cognition and Motor Processes, Springer, Berlin, 1984.

19 Leichnetz, G.R., Afferent and efferent connections of the dorsolateral precentral gyrus (area 4, hand/arm region) in macaque monkey, with comparison to area 8, J. Comp. Neurol., 254 (1986) 460-492. 20 Mufson, E.J. and Mesulam, M.M., Thalamic connections of the insula in the rhesus monkey and comments on the paralimbic connectivity of the medial pulvinar nucleus, J. Comp. Neurol., 227 (1984) 109-120.

21 Petersen, S.E., Robinson, D.L. and Keys, W., Pulvinar nuclei of behaving rhesus monkey: visual responses and their modulation, J. Neurophysiol., 54 (1985) 207-226.

22 Petersen, S.E., Robinson, D.L. and Morris, J,D., Contributions of the pulvinar to visual spatial attention, Neuropsychologia, 25,1A (1987) 97-105.

23 Trojanowsky, J.Q. and Jacobson, S., Medial pulvinar afferents to frontal eye fields in rhesus monkey demonstrated by horseradish peroxidase, Brain Research, 80 (1974) 395-411.

24 Ungerleider, L. and Christensen, C.A., Pulvinar lesions in monkeys produced abnormal eye movements during visual discrimination training, Brain Research, 136 (1977) 189- 196.

25 Ungerleider, L. and Christensen, C.A., Pulvinar lesions in monkeys produced abnormal scanning of a complex visual array, Neuropsychologia, 17 (1979) 493-501. 
26 Wise, S.E, The non-primary motor cortex and its role in the cerebral control of movement. In G. Edelman, W. Cowan and E. Gall (Eds.), Dynamic Aspects of Cortical Functions, Wiley, New York, 1984. 27 Yeterian, E.H. and Pandya, D.N., Corticothalamic connections of the posterior parietal cortex in the rhesus monkey, J. Comp. Neurol., 237 (1985) 408-426.

28 Yirmiya, R. and Hocherman, S., Auditory- and movement-related neural activity interact in the pulvinar of behaving rhesus monkey, Brain Research, 402 (1987) 93-102. 